\title{
Efecto de Surfactantes Polimerizables en la Distribución de Tamaño de Partícula, pH, Viscosidad, Contenidos de Sólidos y de Monómero Residual de una Resina Estireno-butilacrilato
}

\author{
Luis A. Rios, David Ocampo, Alexander Franco, Jhon Fredy Cardona, Fernando Cardeño \\ Universidad de Antioquia, UDEA
}

\begin{abstract}
Resumen: Se presentan los resultados de la polimerización en emulsión de una resina acrílica estirenada usando surfactantes polimerizables, los cuales presentan ventajas técnicas como baja formación de espuma, alta resistencia de la película al contacto con agua y buena estabilidad en la polimerización. Se evaluó el efecto de estos surfactantes polimerizables en la distribución de tamaños de partícula de la resina; además se determinaron propiedades finales de la resina tales como porcentaje de sólidos, porcentaje de monómero libre, viscosidad y pH. Estos resultados se compararon con los obtenidos usando surfactantes convencionales no polimerizables del tipo alquil fenol etoxilado y alquilarilpoliglicol éter sulfato de sodio. Los resultados indican que que se pueden remplazar totalmente los surfactantes convencionales no polimerizables por los surfactantes poliméricos evaluados sin afectar significativamente las propiedades de la resina, mientras que la sustitución parcial de los surfactantes no polimerizables por los polimerizables induce, en algunos casos, a la formación de partículas con mayor tamaño a las presentes en la muestra estándar.
\end{abstract}

Palabras clave: Polimerización, emulsión, surfactantes polimerizables, resinas.

\section{Effect of Polymerizable Surfactants on Particle Size Distribution, pH, Viscosity, Contents of Solids and Residual Monomer of a Styrene-butylacrylate Resin}

\begin{abstract}
Results on the emulsion polymerization of a styrenated acrylic resin using polymerizable surfactants are presented. These surfactants exhibit low foaming, high film strength upon contact with water and good stability in the polymerization. A comparison was made with results for conventional non-polymerizable alkyl aryl polyglycol ether sulphate, sodium salt and nonylphenolethoxylate surfactants. The effect of these polymerizable surfactants on the particle size distribution of the resin was evaluated; in addition to determining the final properties of the resin such as solids content, free monomer content, viscosity and $\mathrm{pH}$. It is feasible to totally replace non-polymerizable surfactants by the polymerizable surfactants evaluated without affecting significantly the properties of the resin, while partial substitution of the non-polymerizable surfactants induces, in some cases, the formation of particles with larger sizes than those in the standard resin.
\end{abstract}

Keywords: Polymerization, emulsion, polymerizable surfactants, resins.

\section{Introducción}

En el campo de los recubrimientos, la tecnología de los polímeros afronta ambiciosos retos para lograr productos con bajos niveles en emisión de compuestos orgánicos volátiles (VOCs), alta durabilidad a la intemperie, rápido curado, buena adhesión a diferentes sustratos y baja sensibilidad a la humedad ${ }^{[1-4]}$. Cada vez son más los procesos que se están encaminando a la producción de polímeros en emulsión, en los cuales se busca obtener una dispersión de los monómeros en un liquido inmiscible con ellos, que generalmente es agua. Uno de los polímeros en emulsión más representativo son las resinas acrílicas estirenadas que en su mayoría son polímeros termoplásticos que tienen excelentes ventajas técnicas, especialmente de durabilidad en exteriores por su alta foto estabilidad y de resistencia a la hidrólisis y a medios ácidos y alcalinos. De igual manera su alta dureza y excelente aspecto de la película las posicionan como una de las resinas de mayor interés en el mercado de polímeros en emulsión. Sus aplicaciones son ampliamente reconocidas en el sector de los adhesivos, recubrimientos y como agentes que incrementan la viscosidad de otros sistemas, dispositivos para la liberación controlada de drogas, inmovilización de enzimas y microorganismos. Su síntesis en fase acuosa ha convertido a esta familia de resinas en productos atractivos por la baja generación de VOCs. Estas resinas se sintetizan a partir del estireno con monómeros acrílicos los cuales son esteres del acido acrílico o metacrílico; algunos de estos ésteres son el metil-, etil-, isobutil-, n-butil-, 2-etilexil-, occil-, lauril-, y estearil-

Autor para correo: Fernando Cardeño, Sede de Investigación Universitaria-Universidad de Antioquia - UDEA 
acrilatos. Estos sistemas pueden generar polímeros con mayores aplicaciones dado que pueden tener otros grupos funcionales como grupos hidroxil (hidroexietil metacrilato), grupos amino (dimetilaminoetil metacrilato) y grupos amida (acrilamida) ${ }^{[5]}$. Las características fisicoquímicas de estas resinas tienen un amplio rango de aplicación como lo son: los recubrimientos decorativos para interiores y exteriores, impermeabilizantes y la formulación de esmaltes, selladores, y barnices.

Estos procesos de polimerización en emulsión generalmente utilizan tensoactivos aniónicos, catiónicos y anfotéricos. Los surfactante aniónicos son los más empleados en este tipo de sistemas; dentro de los cuales están jabones de ácidos grasos, sulfatos y sulfonatos, siendo los más comunes los del tipo alquil fenol etoxilados, los cuales están catalogados como agentes tóxicos y contaminantes de los sistemas acuáticos debido a su baja biodegradabilidad, por lo cual están siendo prohibidos a nivel mundial. En vista de que los tensoactivos tradicionales no son reactivos, estos no se insertan covalentemente al polímero, generando problemas de estabilidad cuando el sistema se somete a ciertas condiciones de fuerza iónica, enfriamiento, agitación o transporte, esto se traduce en migraciones del surfactante hacia las interfases del sistema polimerico. De esta manera se pueden iniciar procesos que afectan la calidad de la película generando problemas con la adhesión sobre el sustrato, alta absorción de humedad y mala estabilidad dimensional. Todo ello se genera debido a la desorción del surfactante desde la partícula de polímero o también migrar desde la superficie de la película formada promoviendo la aparición de defectos o fracturas ${ }^{[6-9]}$. Los surfactantes polimerizables se han planteado como una alternativa que permite el desarrollo de materiales poliméricos con reacciones hibridas a escala nanométrica y con geometrías estables y definidas. Los surfactantes polimerizables son compuestos que aun teniendo la funcionalidad de un tensoactivo convencional son además modificados suministrando en su estructura un grupo funcional con insaturaciones que permite vincularse covalentemente al polímero. Estos grupos funcionales son localizados generalmente al final de la secuencia hidrofóbica mientras que la estructura hidrofílica es compuesta por poli oxido de etileno. Algunos de los grupos funcionales de mayor uso son del tipo alílico, maléico, metacrílico y vinílico ${ }^{[9-16]}$. Debido a que los surfactantes polimerizables quedan fijos en las estructura del polímero, no representan ningún riesgo ambiental.

En este artículo se presentan los resultados de la polimerización en emulsión de una resina acrílica estirenada usando los surfactantes polimerizables comerciales NOIGEN RN-10 ${ }^{\circledR}$ y HITENOL BC-10 ${ }^{\circledR}$; estos resultados se comparan con los obtenidos con los surfactantes no polimerizables tradicionalmente usados como los alquil fenol etoxilado y alquil aril poliglicol éter sulfato de sodio. Se evaluó el efecto de estos surfactantes polimerizables en las propiedades finales de la resina: tamaño de partícula, porcentaje de sólidos, porcentaje de monómero libre, viscosidad y $\mathrm{pH}$.

\section{Experimental}

\section{Materiales y métodos}

Monómeros grado técnico: acrilamida sólida, butil acrilato, estireno, ácido acrílico, reguladores de $\mathrm{pH}$ amoniaco y ácido fórmico, se empleo como biocida formol $(37.7 \%)$. Como tensoactivos no polimerizables se emplearon el Nonil fenol $20 \mathrm{NN}$ (alquil fenol etoxilado de 20 moles de etoxilacion, marca Clariant) y el DISPONIL AES $72^{\circledR}$ (alquil aril poliglicol éter sulfato de sodio, marca COGNIS). Como tensoactivos polimerizables se emplearon NOIGEN RN-10 (Poli oxi-etilen aquil fenil eter) y HITENOL BC-10 ${ }^{\circledR}$ (Poli oxi-etilen alquil fenil eter sulfato de amonio) fabricados por Dai-Ichi Kogyo Seiyaku Co. Ltd. Japon. Además se uso: $\mathrm{NaOH}$ (Sigma-Aldrich), Peróxido de Hidrógeno (35\%, Sigma-Aldrich) y persulfato de potasio (Sigma-Aldrich) como iniciador se empleó.

\section{Sistema de reacción}

Para la preparación de la resina acrílica estirenada se usó un balón de vidrio de 1 litro provisto con tres bocas esmeriladas y divisiones en forma de $\mathrm{Y}$, con el fin de dosificar tanto los catalizadores como la fase monomérica por medio de columnas con llave de teflón (embudos de adición) y para contar con un sistema de reflujo con condensador enfriado con agua, el cual evita la evaporación de monómeros y permite controlar la temperatura de la reacción. Para la fuente térmica se usó una manta de calentamiento con control de temperatura y un dispositivo mecánico del tipo gato mecánico que permite subir y bajar el sistema de reacción.

\section{Procedimiento de síntesis}

Los sistemas de polimerización en emulsión involucran un gran numero de variables; en este trabajo se investigó únicamente el efecto del tipo de tensoactivo; por lo cual se dejaron constantes las demás variables del proceso, las cuales se han determinado en experimentación previa. Se realizan los cálculos para una producción de 800 gramos de resina. Con base en los índices del Balance lipofílico-hidrofílico (HLB), es decir, la medida de la afinidad relativa de los tensoactivos (no iónicos) para las fases acuosa y monomérica ${ }^{[17]}$, se determinó que la cantidad del tensoactivo aniónico debe estar entre 0.5 y $1.5 \%$ de la resina total y la del tensoactivo no iónico entre 0.5 y $1.0 \%$ de la resina total, esto se fundamenta en que para estos sistemas de resinas acrílicas la suma total de los tensoactivos tanto aniónicos como no iónicos no debe superar habitualmente el $2.5 \%$ de la resina total, debido principalmente a aspectos técnicos de desempeño de la resina en la pintura y a valoraciones económicas. En este caso, los tensoactivos no iónicos empleados, Nonil fenol $20 \mathrm{NN}$ y el NIOGEN RN-10 ${ }^{\circledR}$, tienen valores de HLB de 16 y 12.6 respectivamente. Se planteó la siguiente experimentación (Tabla 1), remplazando el DISPONIL AES $72^{\circledR}$ y el Nonil fenol $20 \mathrm{NN}$, parcial y totalmente, por lo tensoactivos polimerizables NOIGEN RN-10 ${ }^{\circledR}$ y HITENOL BC-10. 


\section{Síntesis de resina la acrilica estirenada}

Para el proceso de polimerización en emulsión, se empleó la técnica de la semilla conformada por la mezcla de los monómeros acrilamida, butil acrilato, estireno y acido acrílico con agua y surfactantes (mezcla A), cuya formulación típica se presenta en la Tabla 2 (sin iniciador), la cual se dosifica en un tiempo total de 3 horas y 30 minutos al reactor que contiene la fase acuosa, que corresponde a la mezcla $\mathrm{B}$, tal como se muestra en la Tabla 3. La mezcla A se mantuvo en constante y vigorosa agitación durante todo el tiempo que fue alimentada para mantener su composición constante. Como iniciador de la reacción se emplea persulfato de potasio disuelto en agua y mezclado con tensoactivo aniónico; la concentración del tensoactivo aniónico en esta mezcla (agua-persulfato-tensoactivo aniónico) debe oscilar entre 4-5\%. Esta mezcla que contiene el iniciador se dosifica junto con la mezcla monomérica al reactor que contiene la fase acuosa. Terminado el tiempo de adición tanto de la fase monomérica como del catalizador, se realiza una etapa de pos-polimerizacion con catalizador adicional por tiempo de 1 hora para ajustar el contenido de monómero residual, seguida por una etapa de ajuste sólidos con agua y de $\mathrm{pH}$ con amoniaco y $\mathrm{NaOH}$, finalmente se realiza la adición de agentes biocidas como el formol después del enfriamiento de la mezcla. El proceso se realizó a una temperatura de $78 \pm 2{ }^{\circ} \mathrm{C}$.

Los productos obtenidos se caracterizaron mediante las siguientes técnicas:

- Tamaño de partícula: se empleóla técnica de difracción láser empleando un equipo MASTERSIZER 2000 y con parámetros de índice de refracción de 1.33 y de absorción de 0.001 para las resinas sintetizadas;
- Porcentaje de sólidos: se aplicó la Norma Técnica Colombiana NTC 1774, empleando una estufa MEMMERT;

- Porcentaje de monómero libre: se empleó un cromatógrafo de gases AGILENT 7890A, con detector FID y se aplicó la norma ASTM D4747-08;

- Viscosidad: se aplicó la Norma Técnica Colombiana, NTC 952, estandarizando y empleando un viscosímetro BROOKFIELD; and

- pH: se aplicó la Norma Técnica Colombiana, NTC 1551, empleando un PH-metro PH 3110.

\section{Resultados y Discusión}

La estructura de los surfactantes polimerizables empleados se muestra en la Figura 1; estos surfactantes presentan insaturaciones, que son los grupos químicos polimerizables mediante los cuales se incorporan a la estructura de los polímeros.

En cuanto a los parámetros de viscosidad, monómero libre, $\mathrm{pH}$ y contenido de sólidos se realizaron duplicados los cuales arrojaron valores esperados (véase Tabla 4).

Los parámetros medidos para la viscosidad, contenido de sólidos, monómero libre, $\mathrm{pH}$ y contenido de sólidos no presentan diferencias apreciables que se puedan relacionar con la formulación de los tensoactivos, puesto que son valores típicos esperados de acuerdo a la formulación y proceso realizado para la producción de la resina acrílica.

La propiedad de la resina que tuvo los cambios más significativos fue el tamaño de las partículas. La distribución de tamaños de partículas se muestra en la Figura 2 y en la Tabla 5 se resumen las principales observaciones.

Tabla 1. Condiciones experimentales evaluadas.

\begin{tabular}{|c|c|c|c|c|}
\hline \multirow{4}{*}{ Ensayo } & \multicolumn{4}{|c|}{ Tensoactivos (\%) } \\
\hline & \multicolumn{2}{|c|}{ Aniónico } & \multicolumn{2}{|c|}{ No iónico } \\
\hline & DISPONIL AES $72^{\circledast}$ & *HITENOL BC-10 ${ }^{\circledast}$ & Nonilfenol & *NIOGEN RN-10 ${ }^{\circledR}$ \\
\hline & & & 20 moles & \\
\hline E1 & 1 & 0 & 0 & 1 \\
\hline E2 & 0 & 1 & 1 & 0 \\
\hline $\mathbf{E 3}$ & 0 & 1 & 0 & 1 \\
\hline $\mathbf{E 4}$ & 0.5 & 0.5 & 0.5 & 0.5 \\
\hline E5 & 1 & 0 & 0.5 & 0.5 \\
\hline E6 & 0.5 & 0.5 & 0 & 1 \\
\hline E7 & 0 & 1 & 0.5 & 0.5 \\
\hline E8 & 0.5 & 0.5 & 1 & 0 \\
\hline Estándar & 1 & 0 & 1 & 0 \\
\hline
\end{tabular}

*Tensoactivos polimerizables.

Tabla 2. Fase monomérica (mezcla A).

\begin{tabular}{lc}
\hline \multicolumn{1}{c}{ Reactivos } & $(\boldsymbol{\%})$ \\
\hline Agua desionizada & 15 \\
DISPONIL AES $72^{\circledR}$ o HITENOL BC-10 ${ }^{\circledast}$ & Variable $(0.5-1)$ \\
Acrilamida & 0.8 \\
Butil Acrilato & 24.5 \\
Estireno & 23.5 \\
Acido Acrílico & 1.2 \\
\hline
\end{tabular}

Tabla 3. Fase acuosa.

\begin{tabular}{lc}
\hline \multicolumn{1}{c}{ Reactivos } & $(\%)$ \\
\hline Agua desionizada & 30 \\
NIOGEN RN- $10^{\circledast}$ o Nonil fenol 20 moles & Variable $(0.5-1)$ \\
Ácido fórmico & 0.6 \\
DISPONIL AES $72^{\circledR}$ O HITENOL BC- $10^{\circledast}$ & Variable $(0.5-1)$ \\
Semilla & $4-7$ mezcla A \\
Iniciador & 2 \\
\hline
\end{tabular}


<smiles>CC=Cc1cc(-c2ccccc2)ccc1OC(=O)O</smiles>

NOIGEN RN-10®

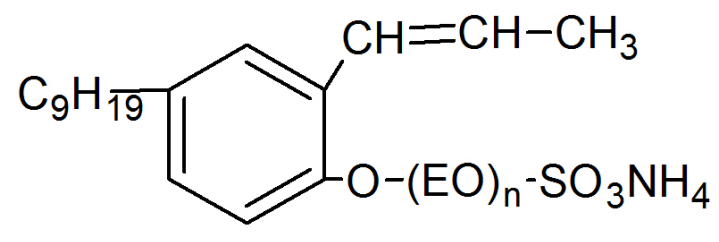

HITENOL BC-10®

Figura 1. Estructuras generalizadas del HITENOL BC- $10^{\circledR}$ y NOIGEN RN-10 ${ }^{\circledR}$.

Tabla 4. Parámetros promedio para todas las resinas.

\begin{tabular}{ccccccccc}
\hline \multirow{2}{*}{ Muestra } & \multicolumn{2}{c}{$\begin{array}{c}\text { Viscosidad } \\
(\mathbf{C p})\end{array}$} & \multicolumn{2}{c}{$\begin{array}{c}\text { Monómero libre } \\
(\boldsymbol{\%} \mathbf{m} / \mathbf{m})\end{array}$} & \multicolumn{2}{c}{$\mathbf{p H}$} & \multicolumn{2}{c}{$\begin{array}{c}\text { Contenido de sólidos } \\
(\% \mathbf{m} / \mathbf{m})\end{array}$} \\
\cline { 2 - 10 } E1 & Valor & Error & Valor & Error & Valor & Error & Valor & Error \\
\hline E2 & 5900 & 100 & 0,24 & 0,01 & 8,1 & 0,1 & 50 & 1 \\
E3 & 6100 & 100 & 0,23 & 0,01 & 8,2 & 0,1 & 49 & 1 \\
E4 & 6100 & 100 & 0,24 & 0,01 & 8,1 & 0,1 & 50 & 1 \\
E5 & 6000 & 100 & 0,23 & 0,01 & 8,1 & 0,1 & 52 & 1 \\
E6 & 5900 & 100 & 0,24 & 0,01 & 8,2 & 0,1 & 51 & 1 \\
E7 & 6000 & 100 & 0,25 & 0,01 & 8,1 & 0,1 & 49 & 1 \\
E8 & 6000 & 100 & 0,25 & 0,01 & 8,2 & 0,1 & 52 & 1 \\
Estándar & 6100 & 100 & 0,24 & 0,01 & 8,1 & 0,1 & 51 & 1 \\
\hline
\end{tabular}

Tabla 5. Tamaño de particular.

\begin{tabular}{ccccc}
\hline \multirow{2}{*}{ Nro Ensayo } & \multicolumn{3}{c}{ Tamaño de partícula $(\boldsymbol{\mu m})$} \\
\cline { 2 - 4 } & $\mathbf{D}(\mathbf{0 . 1})$ & $\mathbf{D}(\mathbf{0 . 5})$ & $\mathbf{D}(\mathbf{0 . 9})$ & \multicolumn{1}{c}{ Observaciones* entre $\mathbf{D}(\mathbf{0 . 9}) \mathbf{y} \mathbf{D}(\mathbf{1 . 0})$} \\
\hline E1 & 0.149 & 0.175 & 0.206 & No se observan tamaños mayores a $0.25 \mu \mathrm{m}$ \\
E2 & 0.150 & 0.177 & 0.212 & Se observan pequeñas cantidades entre 3 y $300 \mu \mathrm{m}$ \\
E3 & 0.148 & 0.174 & 0.205 & No se observan tamaños mayores a $0.25 \mu \mathrm{m}$ \\
E5 & 0.148 & 0.174 & 0.205 & No se observan tamaños mayores a $0.25 \mu \mathrm{m}$ \\
E6 & 0.148 & 0.175 & 0.206 & No se observan tamaños mayores a $0.25 \mu \mathrm{m}$ \\
E7 & 0.152 & 0.179 & 0.219 & Se observan pequeñas cantidades entre 30 y $400 \mu \mathrm{m}$ \\
E8 & 0.151 & 0.180 & 22.25 & Se observan pequeñas cantidades entre 3 y $400 \mu \mathrm{m}$ \\
Estándar & 0.144 & 0.173 & 175.68 & Se observan pequeñas cantidades entre 20 y $600 \mu \mathrm{m}$ \\
\hline
\end{tabular}

*Se muestran los detalles gráficos en la Figura 2.

En el ensayo E3 se intercambiaron completamente los surfactantes convencionales DISPONIL AES $72^{\circledR}$ y Nonilfenol, usados en el ensayo estándar, por los surfactantes polimerizables HITENOL BC-10 ${ }^{\circledR}$ y NIOGEN RN-10 ${ }^{\circledast}$ respectivamente; la distribución de tamaño de partículas (Figura 2 y Tabla 5) indica que no hay cambios apreciables con el uso de estos surfactantes polimerizables, lo que permite plantear que se pueda remplazar totalmente los surfactantes convencionales por los surfactantes poliméricos evaluados.

La sustitución parcial de los surfactantes no polimerizables por los polimerizables induce, en algunos casos, a la formación de partículas con mayor tamaño a las presentes en la muestra estándar. Se presentan los siguientes casos admisibles: i) La sustitución del 50\% de los surfactantes convencionales por los surfactantes polimerizables (ensayo E4) tampoco induce cambios significativos en la distribución de tamaños de partícula de la resina. ii) La sustitución del 50\% y 100\% de Nonilfenol por NIOGEN RN-10 ${ }^{\circledast}$ dejando sin sustituir al DISPONIL AES $72^{\circledR}$, ensayos E5 y E1, es también admisible pues no cambia los tamaños de partícula.

Las sustituciones parciales no admisibles son las siguientes: i) La sustitución completa y parcial de DISPONIL AES $72^{\circledR}$ por HITENOL BC- $10^{\circledR}$ sin sustituir al Nonilfenol, ensayos E2 y E8, induce la formación de partículas de 3-300 $\mu \mathrm{m}$ y $20-600 \mu \mathrm{m}$ respectivamente, que no están presentan en la muestra estándar. ii) la sustitución completa de DISPONIL AES $72^{\circledR}$ y parcial de Nonilfenol (ensayo E7) y la sustitución parcial de DISPONIL AES $72^{\circledR}$ y completa de Nonilfenol (ensayo E6) producen la formación de partículas de 3-400 $\mu \mathrm{m}$ y $30-400 \mu \mathrm{m}$ respectivamente.

Se evidencia que los ensayos 6,7 y 8 tienen distribuciones de tamaño de partícula irregular que se presentan posiblemente por: a) las proporciones de tensoactivos empleados para estos ensayos no logran estabilizar el sistema (es posible que no se llegue a la 

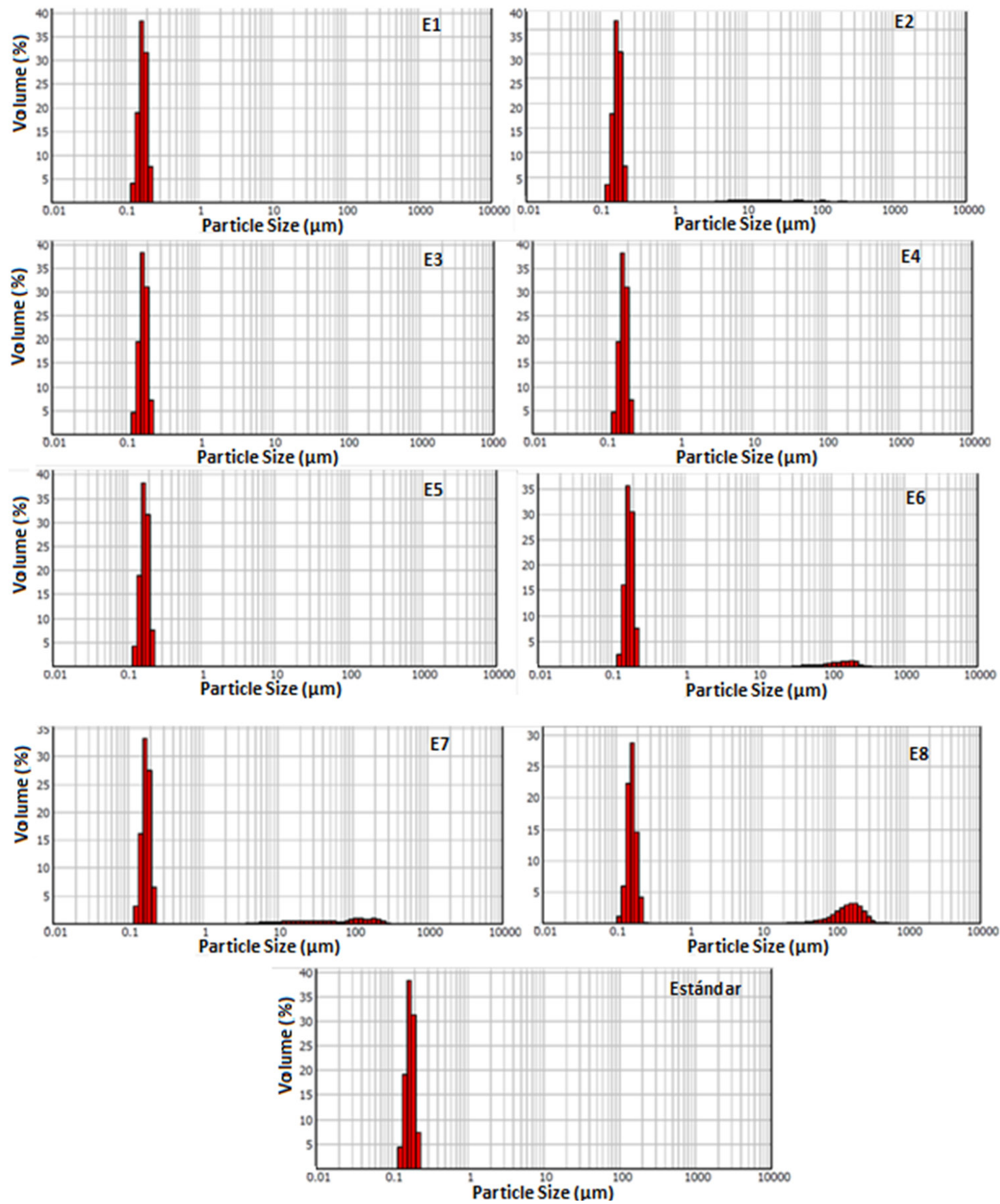

Figura 2. Distribución de tamaño de partícula.

concentración micelar crítica); b) la incompatibilidad de la concentración en el sistema que pueden tener los tensoactivos poliméricos y no poliméricos de acuerdo a las proporciones empleadas para estos ensayos.

\section{Conclusiones}

Las resinas sintetizadas con los surfactantes polimerizables comerciales presentan propiedades fisicoquímicas comparables y muy interesantes para potenciales aplicaciones finales, comparadas con las resinas que emplean surfactantes convencionales. Los surfactantes poliméricos empleados, HITENOL BC-10 ${ }^{\circledR}$ y NIOGEN RN-10 ${ }^{\circledast}$, pueden remplazar en su totalidad a los surfactantes no polimerizables DISPONIL AES $72^{\circledR}$ y Nonilfenol; estos últimos son tóxicos y no se fijan a la resina. La sustitución parcial de los surfactantes no polimerizables por los polimerizables induce, en algunos casos, a la formación de partículas con mayor tamaño a las presentes en la muestra estándar. El trabajo futuro con estos tensoactivos polimerizables sugiere hacer una evaluación complementaria en cuanto a costos y estabilidad de las 
emulsiones en el tiempo y también la evaluación en una formulación y desempeño de una pintura.

\section{Agradecimientos}

Los autores agradecemos a COLCIENCIAS y a la Universidad de Antioquia por su apoyo financiero para llevar a cabo este trabajo.

\section{Referencias}

1. Yu, J. H.; An, J. Y.; Bae, D.; Jung, S.; Kim, S. D.; Ahn, K. S. \& Suh, K. S. - Chem. Mat., 16, p.4693 (2004). http:// dx.doi.org/10.1021/cm049704o

2. Jovanović, R. \& Dubé, M. - Industrial \& Eng. Chem. Res., 44, p.6668 (2005). http://dx.doi.org/10.1021/ie0501778

3. Butler, L. N.; Fellows, C. M. \& Gilbert, R. G. - Prog. Org. Coat., 53, p.112 (2005). http://dx.doi.org/10.1016/j. porgcoat.2005.02.001

4. Zheng, W. J.; Sauer, D. \& Mcginity J. W. - Eur. J. Pharm. Biopharm., 59, p.147 (2005). PMid:15567312. http:// dx.doi.org/10.1016/j.ejpb.2004.06.002

5. Tracton, A. A. - "Coatings Materials And Surface Coatings", cap. 1, p.1 (2007).

6. Šn̆upárek, J.; Bidman, A.; Hanuš, J. \& Hájková, B. - J. Appl. Polym. Sci., 28, p.1421 (1983). http://dx.doi. org/10.1002/app.1983.070280415

7. Swaraj, P. - Prog. Org. Coat., 5, p.79 (1977).
8. Zhao, C. L.; Holl, Y.; Pith, T. \& Lambla, M. - Colloid Polym. Sci., 265, p.823 (1987). http://dx.doi.org/10.1007/ BF01418459

9. Chern, C. S. \& Chen, Y. C. - J. Macro. Sc., Part A, 35, p.965 (1998).

10. Lai, Z.; Sudol, E. D.; Dimonie, V. L. \& El-Aasser, M. S. - J. App. Polym. Sci., p.109 (2008). PMid: 18838950.

11. Asua, J. M. \& Schoonbrood, H. A. S. - Acta Polym., 49, p.671 (1998). http://dx.doi.org/10.1002/(SICI)1521-4044( 199812)49:12<671::AID-APOL671>3.0.CO;2-L

12. Bistline, R. G.; Stirton, A. J.; Weil, J. K. \& Pont, W. S. - J. Am. Oil Chem. Soc, 33, p.44 (1956). http://dx.doi. org/10.1007/BF02638351

13. Holmberg, K. - Prog. Org. Coat, 20, p. 235 (1992).

14. Guyot, A. \& Tauer, K. - Adv. Polym. Sci., 111, p.43 (1994). http://dx.doi.org/10.1007/BFb0024126

15. Guyot, A. - Macromol. Symp., 179, p.105 (2002). http:// dx.doi.org/10.1002/1521-3900(200203)179:1<105::AIDMASY105>3.0.CO;2-7

16. Varela De La Rosa, L.; Sudol, E. D.; El-Aasser, M. S. \& Klein, A. - J. Polym. Sci. Part A: Polym. Chem., 37, p.4073 (1999).

17. Griffin W. C. - J. Soc. Cosmetic Chem., 1, p.311 (1949).

Enviado: $26 / 04 / 12$ Reenviado: $24 / 08 / 12$ Aceptado: 19/09/12 